\title{
Tell Me Again About The Face: Using Repeated Interviewing Techniques to Improve Feature-Based Facial Composite Technologies
}

\author{
Emma Portch \\ Division of Neuroscience and \\ Experimental Psychology, \\ University of Manchester, \\ Manchester, UK
}

\author{
Charlie D. Frowd \\ School of Psychology, \\ University of Central Lancashire, \\ Preston, UK \\ Email: CFrowd1@uclan.ac.uk
}

\author{
Charity Brown \\ School of Psychology, \\ University of Leeds, \\ Leeds, UK \\ Email: psccbr@leeds.ac.uk
}

\begin{abstract}
Facial composite technologies are used to produce visual resemblances of an offender. However, resemblances may be poor, particularly when composites are constructed using traditional 'feature' composite systems deployed several days after the crime. In this case a witness may have forgotten important details about an offender's appearance. Engaging in early and repeated retrieval attempts could potentially overcome this issue. Experiment 1 showed that more recognisable feature composites were produced after participants had provided detailed face recall during two supported retrieval attempts, which included instructions to reinstate the context in which the target had been seen, free recall and cued recall. The first recall attempt was completed on the same day as viewing the target individual, and the second two days later, and immediately before composite construction (traditional forensic procedure). Experiment 2 showed that repeated interviewing only incurred a benefit when the same day interview provided ample retrieval support. The results suggest how traditional forensic procedures can be easily modified to improve the quality of feature composites, and thereby facilitate the detection of offenders.
\end{abstract}

Keywords-facial composites; repeated interviewing; witness; victim; PRO-fit

\section{INTRODUCTION}

A witness or victim to a crime may be asked to construct a facial composite (a visual likeness) of the offender. When circulated among the police force, or wider public, composites that represent a good likeness may be recognised by someone familiar with the individual, providing new investigative leads.

A number of computer-based composite systems are available to law enforcement. These broadly fall into two categories. Traditional 'feature' systems, prevalent in the UK, Europe and USA, emphasise the selection and blending of individual facial features (e.g., E-FIT, PRO-fit, Mac-a-Mug Pro, FACES, Identikit 2000). In contrast, 'holistic', or recognition systems, developed within the UK and South Africa require the witness to select a number of whole faces which are then 'bred' together; an iterative process that continues until a single 'best' face emerges [e.g., EvoFIT, EFIT-V, ID; for a review see 1].
Prior to building a composite with any system, it is good practice to elicit a description of the offender's face from the witness. Cognitive Interviewing techniques (CI) are typically used as they support memory retrieval [2]. In brief, after building a rapport with the witness, the police practitioner asks him or her to think back to the incident, visualise the offender's face (context reinstatement) and describe everything that can be remembered about the face (free recall). The interviewer can then consider asking the witness to focus on each facial feature in turn, and prompt for further recall (cued recall). Eliciting a detailed face description is particularly important when generating feature composites. These systems house an extensive database of exemplar features that the witness can select, and edit for size, position and shade, all within the context of a whole face (also selected by the witness). The witness's description is integral to the procedure as it reduces the pool of alternative features to a manageable size.

Face recall may be limited when the witness experiences a long delay between viewing the offender and providing a description. There is strong evidence to suggest that memory traces, both for faces and events, decay very quickly within the first 24 hours after encoding [3,4]. Although the decay function flattens after this initial period [3], a witness is now more likely to recall coarse-level descriptions (e.g., estimates of age), while 'fine-grained' details about the face, may be lost [5]. Research also highlights a corresponding decrease in feature composite accuracy at long delays. While composites constructed a few hours after a crime tend to be identified reasonably well [17-19\% correct naming rates; 6,7], those constructed after a forensically-relevant delay ( $\geq$ one day) may be poorly recognised $[\leq 5 \% ; 8,9]$. It is reasonable to assume that, following long delays, poor face recall may be an important factor limiting the effective construction of feature composites.

Here, we evaluate the potential for improving feature construction using two techniques that are likely to enhance face recall. Across two experiments, we conduct (1) an early (i.e., same day) face-recall CI and / or (2) a repeated retrieval attempt (i.e., a second $\mathrm{CI}$ ), immediately prior to composite construction. The effectiveness of the resulting composites 
will be compared against those constructed using traditional forensic methods; here the participant undertakes a CI and then constructs a composite, two days after face encoding.

\section{EXPERIMENT 1}

Event and person descriptions contain more accurate information when obtained within an hour of encoding, rather than at delays of upwards of two days [10]. Thus conducting an early interview may improve the level of description provided by the witness, guiding more effective selection of facial features during composite construction. A successful early recall attempt may also increase the likelihood of later recalling that same information $[11,12]$. Initial retrieval may boost the activation of those linked items in memory, hence increasing their accessibility, and protecting against forgetting [i.e., an associative network account; 13]. Of particular relevance, research shows that face recognition benefits from re-accessing an earlier given face description [14]. Here participants demonstrated superior identification of a target face within a line-up when allowed to re-read their initial interview description immediately before viewing the face array. Sporer et al. [14] proposed that re-accessing this description served to cue visual retrieval of the face in memory. Therefore, asking a witness to engage in a second retrieval attempt, close in time to composite construction, may enable him or her to recall earlier provided verbal information.

Mirroring traditional forensic practice, we conducted a face-recall CI prior to composite construction [9]. We manipulated the timing of the initial CI in relation to when the target face was encoded (same day vs. two days later) as well as including a condition where participants undertook a repeated $\mathrm{CI}$, immediately before composite construction. We expected to find improvements in composite accuracy both as a result of an early, initial retrieval attempt and a repeated retrieval attempt.

\section{A. Method}

We used a two-stage methodology [9]. In Stage 1, a group of participants were each shown a target face from a specific pool of identities (i.e., football players). They then described the face and constructed a feature composite (see Fig. 1). These participants were unfamiliar with the target pool (i.e., non-football fans). In Stage 2, a second group of participants, this time familiar with the target identities (i.e., football fans), attempted to name the composites. Although a forensicallyrelevant procedure, spontaneous naming rates are typically low for composites constructed after two days [8-9], which may limit the sensitivity of this measure for detecting differences in composite quality. Therefore, to boost naming rates we use an additional cued procedure, during which participants were provided with information to constrain the pool of target identities [8].

\section{1) Stage 1: Composite Construction}

\section{a) Participants}

Forty non-football fans (35 females), aged 18-24 years ( $M$ $=20.65, S D=1.51)$, were recruited from a UK university campus.

\section{b) Materials}

Ten good quality head and shoulder colour photographs of male UK footballers were compiled. The targets were in fullface pose with a neutral expression and printed individually on white A4 paper $(8 \mathrm{~cm} \times 9 \mathrm{~cm})$. Composites were constructed using PRO-fit (version 3.1)

\section{c) Design}

Participants were randomly assigned to create a single composite under one of four interview conditions: (1) 2 day CI and construction; (2) same day CI, 2 day construction; (3) same day CI, 2 day CI, and construction; and (4) same day CI and construction. The 10 target identities were re-used once in each between-subjects condition.

\section{d) Procedure}

Participants studied a photograph of an unfamiliar target face for 1 minute in the knowledge that they would later construct a composite of this individual. One group of participants were asked to return two days later to undertake a CI (described below) and construct a composite (2 day CI and construction; traditional forensic practice). Three other groups of participants returned on the same day as viewing the target photograph (3-4 hours later) to undertake a CI. Following the same day CI, one group immediately constructed a composite (same day CI and construction); one group returned two days later to construct a composite (same day CI, 2 day construction); and one group returned two days later to engage in a second CI and construct a composite (same day CI, 2 day CI, and construction). The CI followed the four-stage procedure described in the introduction (rapport building, context reinstatement, free recall and cued recall).

Participants' worked alongside the experimenter to construct a composite. The experimenter first entered the participant's description into PRO-fit to obtain a pool of about 20 examples per facial feature. An "initial" composite, whose appearance matched the description, was then displayed. The composite was re-worked by exchanging, repositioning and resizing features, adjusting brightness/contrast and adding artwork (e.g., stubble) until the participant confirmed that the best possible likeness had been achieved. The entire procedure took about an hour including debriefing.
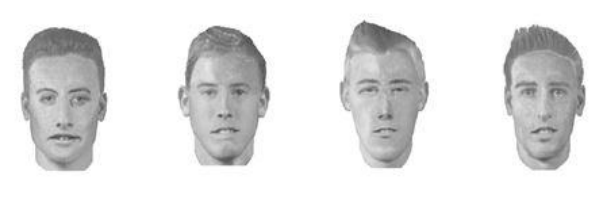

FIG. 1. Example composites of premiership footballer Peter Crouch, constructed (Stage 1) and named (Stage 2) in Experiment 1. Composites were constructed under one of four interview conditions (from left to right): (a) 2 day CI and construction; (b) same day CI, 2 day construction; (c) same day CI, 2 day CI, and construction, and (d) same day CI and construction. 


\section{2) Stage 2: Composite Evaluation (Naming)}

\section{a) Participants}

Participants were 60 football fans (59 male), aged 18-65 years $(M=24.7, S D=9.2)$, from a UK university campus.

\section{b) Materials}

The 40 composites were printed individually in grayscale $(7 \mathrm{~cm} \times 10 \mathrm{~cm})$. A front-view colour photograph of each of the 10 footballers was also required. The composites were divided into five sets of eight; each set contained two composites that had been constructed under each of the four interview conditions, and only one example of a single target identity.

\section{c) Design and Procedure}

Participants were tested individually and randomly allocated to view a single composite set with equal sampling $(n=12)$. They viewed a composite set twice, first under spontaneous and then under cued naming conditions, resulting in a 4(Interview) x 2(Naming Task) repeated-measures design. To restrict the pool of potential target identities, participants were told that the composites were constructed to resemble footballers currently playing in the UK premiership who had played for England. Participants viewed the eight composites sequentially (in a different random order for each person) and attempted to spontaneously name each, or gave a "don't know" response. To check that participants were familiar with the target identities, they were then asked to provide a name for each of the eight corresponding target photographs. Next, participants viewed the composites again. Here knowledge of the corresponding pool of targets acted as a cue to composite identity (i.e. 'cued' naming). The naming procedure took about 15 minutes, including debriefing.

\section{B. Results}

Participants were very familiar with the relevant identities, correctly naming the target photographs with a mean of $96.0 \%$ $(S D=19.5 \%)$. A composite was unlikely to attract a correct name if the corresponding target photo was not correctly named. In these relatively few cases, responses (38/960) were treated as missing data and not subject to further analysis.

\section{1) Correct Naming}

Responses to composites were either correct (coded as 1) or incorrect (i.e, a mistaken name or "don't know" response; coded as 0 ). The proportion of correct naming responses are shown in Table I. Mean correct naming was 39.7\% overall $(S D=49.0 \%)$. Throughout, Binary Logistic Regression with Generalized Estimating Equations (GEE) was used to accommodate analysis of dichotomous responses ( 0 and 1) collected in a Repeated Measures design. We use this approach to provide a powerful, unified by-participants and by-item model. Interview, Naming Task, and Target items were coded for the GEE as within-participants variables. An Independent structure was used for the Working Correlation Matrix (which assumes that repeated items were uncorrelated); a Model-based (cf. Robust-) estimator was selected for the Covariance Matrix (as this provides a model fit with lower $S E$ parameters values). The statistical technique involves ChiSquare, and so a check was made to confirm that all $f$ (observed) $>0$, and all $f$ (expected) were not less than 5 for $>20 \%$ of cells.

Once a model was built, the associated $B$ and $S E(B)$ values were checked to be within sensible limits (i.e., with values that were neither too low nor too high). To start, we entered both repeated measures variables into a saturated model: Interview (coded as $1=2$ day $\mathrm{CI}$ and construction; $2=$ same day $\mathrm{CI}, 2$ day construction; 3 = same day $\mathrm{CI}, 2$ day $\mathrm{CI}$, and construction; 4 = same day CI and construction) and Naming Task (coded as $1=$ spontaneous and; 2 = cued), along with their associated two-way interaction. The lowest coded category was selected as the reference as, based on previous research, both the traditional interview method (2 day $\mathrm{CI}$ and construction) and the spontaneous naming condition were expected to elicit lower composite naming. The resulting GEE was re-run removing the interaction term as it contributed least to the variance within naming (Wald $\chi^{2}, p>.10$ ).

The final GEE model revealed Naming Task as a reliable factor $\left[\chi^{2}(1)=64.00, p<.001\right]$. Composites were better named under cued $(52.7 \%)$ than spontaneous naming $(26.7 \%)$ conditions [Slope $B=1.14, S E(B)=0.14$, Odds Ratio $\operatorname{Exp}(B)$ $=3.11,95 \%$ CI $(2.36,4.11)]$. Interview was also significantly associated with correct naming $\left[\chi^{2}(3)=12.13, p=.007\right]$. Compared to composites constructed under the traditional method (2 day CI and construction), composites constructed with an additional same day CI (same day CI, 2 day CI, and construction) elicited significantly more correct names $[B=$ $0.69, S E(B)=0.20, p=.001, \operatorname{Exp}(B)=2.00(1.35,2.96)]$.

In addition, when administering a single CI prior to construction, there was a marginally-significant trend toward better naming rates when the CI was undertaken on the same day as target encoding (followed by construction two days later), than when a traditional method was followed. That is, where both the CI and composite construction took place two days after target encoding $[B=0.39, S E(B)=0.20, p=.05$, $\operatorname{Exp}(B)=1.48(0.99,2.19)]$.

TABLE I. Percentage of composites correctly named (Experiment 1).

\begin{tabular}{|c|c|c|c|c|}
\hline \multirow[b]{3}{*}{$\begin{array}{l}\text { Naming } \\
\text { Task }\end{array}$} & \multicolumn{4}{|c|}{ Interview } \\
\hline & $\begin{array}{c}2 \text { day CI } \\
\text { and } \\
\text { construction } \\
\text { [Traditional } \\
\text { method] }\end{array}$ & $\begin{array}{c}\text { Same day } \\
\text { CI, } \\
2 \text { day } \\
\text { construction }\end{array}$ & $\begin{array}{c}\text { Same day CI, } \\
2 \text { day } C I \\
\text { and } \\
\text { construction }\end{array}$ & $\begin{array}{c}\text { Same day CI } \\
\text { and } \\
\text { construction }\end{array}$ \\
\hline & & & & \\
\hline Spontaneous & $\begin{array}{c}20.9 \\
(24 / 115)\end{array}$ & $\begin{array}{c}25.0 \\
(29 / 116)\end{array}$ & $\begin{array}{c}34.5 \\
(40 / 116)\end{array}$ & $\begin{array}{c}26.3 \\
(30 / 114)\end{array}$ \\
\hline Cued & $\begin{array}{c}43.5 \\
(50 / 115)\end{array}$ & $\begin{array}{c}56.0 \\
(65 / 116)\end{array}$ & $\begin{array}{c}60.3 \\
(70 / 116)\end{array}$ & $\begin{array}{c}50.9 \\
(58 / 114)\end{array}$ \\
\hline Total & $\begin{array}{c}32.2^{\dagger \mathrm{i}} \\
(74 / 230)\end{array}$ & $\begin{array}{c}40.5^{\mathrm{i}} \\
(94 / 232)\end{array}$ & $\begin{array}{c}47.4^{\dagger} \\
(110 / 232)\end{array}$ & $\begin{array}{c}38.6 \\
(88 / 228)\end{array}$ \\
\hline
\end{tabular}

a.Values represent percentage-correct naming rates and are calculated from responses in parentheses: summed correct responses (numerator) and total (correct and incorrect) responses (denominator). These data are for composites for which participants correctly named the relevant target $(N=922$ out of 960$)$. GEE model parameters: [QIC: 1174.52 ; intercept $B=-1.37, S E(B)=0.17, \mathrm{p}<.001,1 / \operatorname{Exp}(B)=3.94$ $(2.83,5.49)]$. $\dagger$ Pairwise contrast significant at $p<.05{ }^{\mathrm{i}} p=.05$ 
The data show no significant advantage of a same day CI when composites were also constructed on the same day that the target face was encoded (same day CI and construction vs. 2 day CI and construction, $p=.14$ ). However, Table I shows a numerical advantage for this condition in proportional naming rates. To increase the statistical power within our design we combined both conditions that included a single $\mathrm{CI}$ on the same day as encoding (same day CI, 2 day construction and same day $\mathrm{CI}$ and construction) and re-ran the GEE. The resulting model showed that engaging in a single $\mathrm{CI}$, on the same day as encoding, led to composites that were correctly named reliably more often $(39.6 \%)$ than when using the traditional construction method (2 day CI and construction; $32.2 \%):[B=0.35, S E(B)=0.18, p=.050, \operatorname{Exp}(B)=1.41$ $(1.00,2.00)]$.

\section{2) Mistaken Naming}

Composites that elicit a high level of mistaken (wrong) names may increase false investigative leads. The number of mistaken names were considered relative to the total number of incorrect names (mistaken, coded as 1; and "don't know" responses, coded as 0$)$. Correct responses $(N=366)$ were removed from the analysis and treated as missing data, as were composites for which the corresponding target had not been correctly named $(N=38)$. Mistaken naming accounted for $78.4 \%$ of incorrect responses.

Again, the GEE commenced with a saturated model and predictors were subjected to sequential removal for those that contributed least to the variance within naming $(p>.10)$. The final GEE model [QIC: 579.94, $B=1.04, S E(B)=0.12, p<$ $.001, \operatorname{Exp}(B)=2.84(2.23,3.62)]$ revealed Naming Task to be a significant predictor of mistaken naming $\left[\chi^{2}(1)=9.86, p=\right.$ $.002, B=0.72, S E(B)=0.23, \operatorname{Exp}(B)=2.05(1.31,3.20)]$ : More mistaken names were elicited in the cued naming $(85.3 \%)$ vs. spontaneous naming $(74.0 \%)$ task.

\section{Discussion}

Analyses for spontaneous and cued naming data suggest that face construction benefits from early retrieval. When participants encountered a single CI, composites were more often correctly named when face recall had been attempted on the same day as face encoding, rather than two days later (a typical forensic delay). The benefit of an early retrieval attempt was apparent whether composites were constructed immediately after the same day interview or two days later. However, the most robust benefit was observed when the early retrieval attempt was supplemented by a subsequent recall attempt, made two days later and immediately before composite construction. The data also reveal that these benefits were not off-set by an increase in mistaken naming.

\section{EXPERIMENT 2}

Results from Experiment 1 suggest that composite construction benefits from an early, same day face recall from memory. Early retrieval attempts may strengthen the representation of facial features in memory, protecting these details from subsequent forgetting [10]. A repeated retrieval attempt, conducted close in time to composite construction, may increase access to this information, boosting face memory via a process of a context reinstatement [14].

Despite the importance of obtaining a thorough face description, research suggests that police officers rarely conduct early, supported retrieval attempts, such as a (full) CI, perhaps due to a lack of officer resources [15-16]. Here we examine the practical question of whether a repeated interviewing advantage would still exist when we use a less time-consuming method to elicit an early description: in this case, a simple free-recall instruction [cf. (full) CI].

In the present task two groups of participants received an early (i.e., same day) interview; one group received a facerecall CI (including context-reinstatement, free recall and cued recall instructions), whereas the other received only free-recall instructions. All three groups of participants returned two days later to complete a CI followed by composite construction. Overall, the naming rates for composites constructed by participants in the two repeated interviewing conditions were compared against those constructed using a traditional forensic method (as in Experiment 1). Target identities were characters from the BBC TV soap EastEnders.

\section{A. Method}

\section{1) Stage 1: Composite Construction}

\section{a) Participants}

Thirty non-EastEnders fans (21 female) aged from 21-25 years $(M=21.82, S D=1.53)$ were recruited from a UK University campus.

\section{b) Materials}

Ten nonviolent video clips from the TV soap EastEnders were used as targets. Each clip portrayed a social interaction between two characters that lasted between 15 and 45 seconds. The final frame of each clip froze on a target individual for 5 seconds. Ten target identities were used: five male and five female characters. Composites were again produced using PRO-fit.

\section{c) Design}

Participants were randomly assigned to create a single composite under one of three interview conditions: (1) 2 day CI and construction; (2) same day Free Recall, 2 day CI, and construction; and (3) same day CI, 2 day CI, and construction. Each of the 10 target identities were used only once in each of these conditions to create a total of 30 composites.

\section{d) Procedure}

Participants viewed one of the ten video clips in the knowledge that they would later construct a composite of the target individual. Two groups then returned 3-4 hours later to complete either a CI or free recall of the target face. Those assigned to the traditional construction method did not attend a same day interview. All participants returned two days later to undertake a CI and composite construction. 


\section{2) Stage 2: Composite Evaluation}

\section{a) Participants}

Forty EastEnders fans (25 female), aged 21-51 years $(M=$ $32.55, S D=9.83$ ) were recruited from two different UK university campuses.

\section{b) Materials}

The composites were printed individually in grayscale $(10 \mathrm{~cm} \times 15 \mathrm{~cm})$. A front-view colour photograph of each EastEnders target identity was also required. The 30 composites were divided into 5 sets of 6 composites; each set contained two composites constructed under each of the three interview conditions, and only one example of a single target identity. Participants were randomly allocated to view a single set $(n=8$, per set $)$.

\section{c) Design and Procedure}

Participants named composites in spontaneous and cued tasks within a 3(Interview) x 2(Naming Task) Repeated Measures design. The procedure followed Experiment 1: spontaneous naming of composites, spontaneous naming of photographs and cued naming of composites.

\section{B. Results}

Participants showed high target familiarity, correctly naming the target photographs $94.0 \%(S D=23.5 \%)$ of the time. As in Experiment 1, composites corresponding to unknown targets were treated as missing data (14/240).

\section{1) Correct Naming}

Mean correct naming across composite items was $64.4 \%$ ( $S D=47.9 \%$, see Table II for a summary). As before, we began with a saturated model, entering both repeated measures variables: Interview (coded as $1=2$ day $\mathrm{CI}$ and construction; 2 = same day Free Recall, 2 day CI, and construction; 3 = same day CI, 2 day CI, and construction) and Naming Task (coded as $1=$ spontaneous; and 2 = cued) along with their associated two-way interaction. The lowest coded category was selected as the reference.

Based on the outcome of Experiment 1 it was expected that lower naming would result from:

TABLE II. Percentage of composites correctly named (Experiment 2)

\begin{tabular}{lccc} 
& \multicolumn{3}{c}{ Interview } \\
\cline { 2 - 4 } & $\begin{array}{c}\text { 2 day CI and construction } \\
\text { [Traditional } \\
\text { Method] }\end{array}$ & $\begin{array}{c}\text { Same day Free Recall, } \\
\text { day CI and construction }\end{array}$ & $\begin{array}{c}\text { Same day CI, 2 } \\
\text { day CI and } \\
\text { construction }\end{array}$ \\
\cline { 2 - 4 } Naming Task & $32.4^{\dagger}$ & & \\
Spontaneous & $(24 / 74)$ & $43.2^{\mathrm{i}}$ & $57.7^{\dagger} \mathrm{i}$ \\
& & $(32 / 74)$ & $(45 / 78)$ \\
Cued & 86.1 & & 85.5 \\
& $(62 / 72)$ & $(60 / 73)$ & $(65 / 76)$ \\
Total & & & 71.4 \\
\hline
\end{tabular}

$b$. See Table I. These data are for composites for which participants correctly named the relevant target $(N=447 / 480)$. GEE model parameters: $[$ OIC: 503.43; $B=-0.73, S E(B)=0.25, p=.003,1 / \operatorname{Exp}(B)=$ $2.08(1.28,3.39)]$. $\dagger$ Pairwise contrast significant at $p<.05,{ }^{\mathrm{i}} p<.08$ (a) the traditional forensic method (2 day CI and construction) versus the other interview conditions, and (b) spontaneous versus cued naming conditions.

The resulting GEE was re-run without the main effect of Interview as it contributed least to naming variance $(p>.10)$. The final GEE model revealed Naming Task as a reliable factor $\left[\chi^{2}(1)=69.95, p<.001\right]$ with composites eliciting more correct names in the cued $(84.6 \%)$ than spontaneous naming task $(44.7 \%)$. The main effect was qualified by an Interview $\times$ Naming Task interaction $\left[\chi^{2}(4)=10.17, p=.038\right]$. The interaction was driven by differences in naming rates across interview conditions in the spontaneous, but not cued naming task (all $p s>.50$ for the latter comparisons). For spontaneous naming, composites constructed with an additional same day CI (same day CI, 2 day CI, and construction) elicited significantly more correct names than those constructed using a traditional method (2 day $C I$ and construction): $[B=1.04$, $S E(B)=0.34, p=.002, \operatorname{Exp}(B)=2.84(1.47,5.51)]$. The benefits of engaging in an early interview did not arise when the initial retrieval involved only free recall. Here composites were of similar quality to those constructed under a traditional method ( $p=.18)$. Consistent with this, there was a trend (marginally significant) towards better naming for composites constructed after a same day CI as opposed to same day free recall $[B=0.58, S E(B)=0.33, p=.08, \operatorname{Exp}(B)=1.79(0.94$, 3.40)].

\section{2) Mistaken Naming}

As before, incorrect responses included mistaken names (coded as 1) and "don't know" responses (coded as 0). Correct responses $(N=313)$ were removed from the analysis and treated as missing data, as were composites for which the corresponding target was not correctly named $(N=14)$. Mistaken naming accounted for $50.3 \%$ of incorrect responses. GEE revealed no significant predictors for this DV $(p>.10)$.

\section{Discussion}

Replicating Experiment 1, the present findings showed that, when a CI and composite construction take place two days after a witnessed event (the traditional method), composite quality can be improved by including an additional early CI. This procedure thus improved accurate naming of composites, without a corresponding increase in mistaken naming. The finding generalised to a new set of target faces and a different presentation format at encoding (i.e., video stimuli vs. static photographs). However, early face recall only provided an advantage when it was obtained using adequate retrieval support $[11,12]$. When participants attempted early retrieval under free recall instructions they constructed composites that attracted marginally fewer correct names than those constructed following an early face-recall CI. More importantly, composites constructed after an early free recall attempt were of no better quality than those constructed using a traditional method (i.e., where no early recall is elicited).

Similar to Experiment 1, composites were correctly named more often in the cued than spontaneous naming task. Here, providing participants with information about target, and thus composite identity, produced near-ceiling naming levels. Typically, some cues will be provided alongside composites 
circulated as part of an appeal, which may narrow the pool of people considered by potential recognisers (e.g., age, build, weight). In forensic situations then, access to additional cues may well increase the potential that a composite is named.

\section{GENERAL DiscuSSION}

The current work demonstrates that witnesses' ability to construct a feature composite depends on conditions that support their memory of a target face. First, an early recall attempt may aid composite construction by maintaining important visual details and protecting those details from subsequent forgetting [10]. Second, these benefits may be secured through a subsequent recall attempt, which occurs close in time to composite construction [14]. The current research highlights an important caveat: the benefit of an early retrieval attempt only occurs when using a technique that provides ample retrieval support [11,12]. Indeed, while retrieval elicited via an additional early CI (including context reinstatement, free and cued recall) improved composite quality, simple free recall did not.

Theoretically, associative models of memory would predict benefits after engaging in retrieval shortly after face encoding. These models view episodic memory as a network, encompassing items and the links between them, with retrieval serving to boost the activation of both components [13]. Thus, early face recall may increase the activation of items representative of how the face is encoded in memory, helping to maintain that information over time [10]. However, information in memory is more likely to be successfully maintained if early recall is adequately supported. Undertaking a face-recall CI immediately prior to composite construction following an earlier (same day) CI, but not free recall attempt, produced more recognisable composites. Here, the second interview may have acted to facilitate face construction by successfully cueing visual information, originally encoded about the target face, thereby creating context reinstatement [14]. However, context reinstatement may only be of benefit when the original encoded memory has been adequately strengthened through earlier supported retrieval, in this case via an early face recall CI.

In conclusion, feature composite systems come with an inherent practical constraint: the witness must be able to provide a sufficiently detailed description of the relevant face. Without this description, a manageable pool of individual features cannot be created and composite construction is inadvisable [17]. In current practice, witnesses usually encounter a post-event delay of at least two days before they provide a description of the offender (using a CI) and construct a composite. During this delay, memory for the offender's face may be subject to forgetting, or contamination, from information introduced by other sources [e.g., cowitnesses; 18]. Providing witnesses with an additional opportunity to engage in supported face-recall (CI), preferably on the same day as they witness the crime, can improve subsequent composite accuracy. A minor adaptation to current practice may provide an appropriate opportunity for an early retrieval. It may be possible for police practitioners to conduct an early CI over the telephone: a conversation usually undertaken to build rapport with the witness and invite them to a subsequent interview and composite construction session. Our experimental findings showed that an additional same day CI more than doubled naming rates compared to the traditional method $[\operatorname{Exp}(B)=2.84]$, suggesting that this procedure will bring a worthwhile benefit to the effectiveness of composites when implemented within current policing procedures.

\section{ACKNOWLEDGMENT}

We thank Sophie Marsh-Picksley and Ed Bamforth for contributing to data collection.

\section{REFERENCES}

[1] C. D. Frowd et al. "A decade of evolving composites: regression- and meta-analysis", Journal of Forensic Practice, 2015,17(4), 319 -334.

[2] R. E. Fisher, and R. E. Geiselman, R. E. "Memory enhancing techniques for investigative interviewing: The cognitive interview". Springfield, IL: Thomas, 1992.

[3] H. Ebbinghaus. "Über das Gediichtnis [Memory]". Leipzig: Duncker and Humblot, 1885.

[4] P. N. Shapiro, and S. Penrod. "Meta-analysis of facial identification studies", Psychological Bulletin, 1986, 100(2), 139-156.

[5] M. Goldsmith, A. Koriat, and A. Pansky. "Strategic regulation of grain size in memory reporting over time", Journal of Memory and Language, 2005, 52(4), 505-525.

[6] G. Davies, P. van der Willik, and L.J. Morrison. "Facial composite production: A comparison of mechanical and computer-driven systems", Journal of Applied Psychology, 2000, 85(1), 119-124.

[7] C. D. Frowd et al. "Contemporary Composite Techniques: the impact of a forensically-relevant target delay", Legal and Criminological Psychology, 2005, 10, 63-81.

[8] C. D. Frowd, and S. Fields. "Verbalization effects in facial composite production". Psychology, Crime and Law, 2011, 17(8), 731-744.

[9] C. D. Frowd et al. "A forensically valid comparison of facial composite systems". Psychology, Crime and Law, 2005, 11(1), 33-52.

[10]E. B. Ebbesen, and C. B. Rienick. "Retention interval and eyewitness memory for events and personal identifying attributes", Journal of Applied Psychology, 1998, 83, 745-762.

[11] F. Gabbert, L. Hope, and R. P. Fisher. "Protecting eyewitness evidence: Examining the efficacy of a self-administered interview tool", Law and Human Behavior, 2009, 33(4), 298-307.

[12] L. Hope, F. Gabbert, R. P. Fisher, and K. Jamieson. "Protecting and enhancing eyewitness memory: The impact of an initial recall attempt on performance in an investigative interview", Applied Cognitive Psychology, 2014, 28(3), 304-313.

[13] J. R. Anderson. "A spreading activation theory of memory", Journal of Verbal Learning and Verbal Behavior, 1983, 22(3), 261-295.

[14] S. L. Sporer, K. S. Kaminski, M. C. Davids, and D. McQuiston. "The verbal facilitation effect: re-reading person descriptions as a system variable to improve identification performance", Memory, 2016, 24(10), 1329-1344.

[15] M. R. Kebbell, and R. Milne. "Police officers' perceptions of eyewitness performance in forensic investigations", The Journal of Social Psychology, 1998, 138(3), 323-330.

[16] C. Brown, T. J. Lloyd-Jones, and M. Robinson. "Eliciting person descriptions from eyewitnesses: A survey of police perceptions of eyewitness performance and reported use of interview techniques", European Journal of Cognitive Psychology, 2008, 20(3), 529-560.

[17] Association of Chief Police Officers (ACPO). "Facial Identification Guidance", retrieved from http://www.acpo.police.uk/documents/crime/2009/200911CRIFIG01.pdf , 2009.

[18] M. S. Zaragoza, and S. M. Lane. "Source misattributions and the suggestibility of eyewitness memory". Journal of Experimental Psychology: Learning, Memory, and Cognition, 1994, 20(4), 934-945. 